\section{Teeth and their Associated Tissues}

Barry R. J. Rittman

University of Texas-Houston Health Science Center

The preparation of histological sections of teeth can be both a rewarding and a frustrating experience. This is primarily due to the varying degrees of mineralization of the enamel, dentin, cementum and surrounding bone and the difficulty in retaining original relationships between the calcified and the soft tissues in the final stained section.

The tooth has a central pulp that is a gelatinous loose connective tissue. Surrounding this is dentin that forms the main bulk of the tooth, is composed chiefly of collagen fibers and is approximately $70 \%$ mineralized. The crown is covered with enamel, a brittle material that is approximately $96-97 \%$ mineral. Covering the root is a thin layer of cementum similar to bone in its mineralization (approximately $50 \%$ ). The tooth in situ is held in a bony socket by a collagenous periodontal ligament, with the ends of its collagen bundles inserting into both the bone and the cementum of the root. The surrounding bone is a mixture of spongy bone surrounded by outer plates of compact bone.

One question frequently asked is why would anyone wish to carry out histology on a tooth that is obviously no longer of use to a patient. The answer lies in the wealth of knowledge that may be obtained by examining genetic imperfections, studying the process of remineralization, evaluating new methods for the regeneration of gingiva, and optimizing techniques for the retention of dental implants. Studies are carried out on the structure of enamel, dentin and cementum, patterns of growth during development, comparison of deciduous and permanent tooth development and factors which affect tooth eruption, occlusion, retention of teeth and tooth movement. Continuously erupting teeth such as rodent incisors are often used as models to study the development of the dental tissues as these teeth show cells in all stages of development.

Preparation of tooth sections is generally accomplished by one of two procedures. In the preparation of ground sections, the enamel is retained. In the other, processing involves removal of the mineral component so that tissue can be processed to paraffin wax and sections prepared. During demineralization, the enamel will fragment and is lost. These two general techniques complement each other in the information that they can provide.

Ground sections are prepared by slicing the tooth with an abrasive disc or diamond impregnated saw. During this process, thin slices in the order of $100-200 \mu \mathrm{m}$ are cut but a considerable amount of the tissue between each section is lost. Sections may then be made thinner by grinding each surface with diamond powders or abrasive papers. With care, sections in the 5 to $10 \mu \mathrm{m}$ range can be prepared, examined and stained. Ground sections are useful for examining defects in enamel and for general histology after appropriate staining but their preparation requires considerable technical expertise and soft tissues are lost. Thick ground sections can be used to examine carious lesions, orientation of dentinal tubules, retention of filling material, leakage around fillings, implanted materials in bony sockets etc.

A variety of techniques such as Nomarski Differential Interference Contrast can be used for transmitted light microscopy and confocal microscopy can be used to visualize structures at different optical planes in the sections. Using these techniques it is

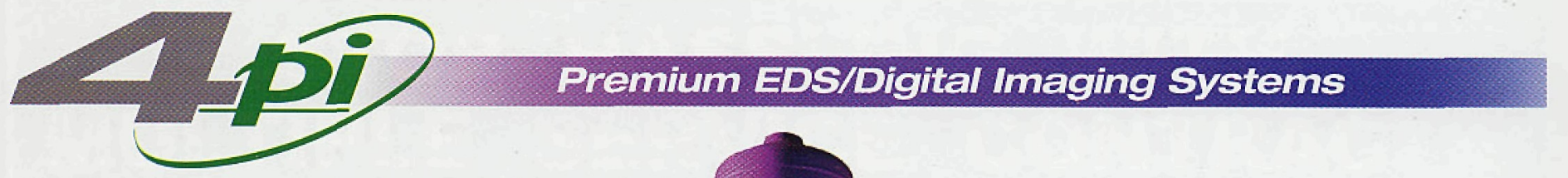

4pi Analysis provides EDS and digital imaging systems, components, and software for both the MaC ${ }^{\oplus} \mathrm{OS}$ and Windowse 98 or NT. Whether you need a complete system or want to upgrade outmoded electronics, 4 pi's products meet the high standards you demand.

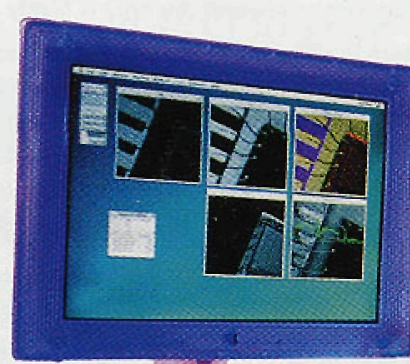


possible to follow the course of dentinal tubules and growth markers in sections up to several hundred micrometers thick. Reflected light microscopy may also be useful. Staining the surface with toluidine blue, can provide a great deal of histologic detail as the stain generally only penetrates a few micrometers. The stained surface can also be reground to examine additional features that are deeper in the block.

Several methods exist to examine dentinal tubules. A common technique is to remove the pulp, place the tooth in India ink and subject to vacuum. Dentinal tubules are generally filled by this technique. Another technique is Colquhoun's method in which ground sections are soaked in mercuric chloride and then placed in ammonium sulfide. A fine deposit of mercuric chloride fills all spaces including the dentinal tubules. Thinner sections can then be prepared by grinding on abrasive papers.

A major problem with ground sections is the loss of soft tissues during the procedure. If soft tissues are to be retained then the tooth must be processed with a plastic such as methyl methacrylate. Sections may be cut with an abrasive disc and ground thinner using abrasive papers as before. Soft tissues are retained but the plastic embedding medium, unless removed, provides an impermeable barrier to the penetration of many dyes. Additionally, plastic sections tend to curl when they are ground down below a certain thickness. This curling can be minimized by grinding one side and then cementing this face to a plastic or glass slide before completing the grinding.

Fixation can be by perfusion but care must be taken that pressure is carefully controlled. Since the pulp is surrounded by a rigid chamber, excessive pressure is not easy to dissipate and may cause damage to the pulpal vessels. Penetration of agents into the pulp is slower than into bone and this may be improved by removing the apex of the tooth.

Developing teeth have a lower mineral content especially in forming enamel and can often be sectioned without subjecting them to demineralization. Attempts at a partial demineralization generally result in the enamel becoming fragmented and lost. Preferred methods of demineralization include disodium EDTA, sodium formate, and formic acid. EDTA with polyvinyl pyrrolidone appears to work well with many immunochemical techniques. With acid demineralization, tissues must be in a solution that is constantly circulating to prevent saturation of the solution with mineral and reprecipitation of mineral in the hard tissues. Round amorphous deposits of mineral appear to be deposited chiefly in dentin and once formed cannot be removed. Detecting the completeness of demineralization such methods as chemical end point, bending, slicing a portion off the tooth or probing with a needle are not advisable. X-rays are the preferred method.

While this decalcification process removes mineral, it usually does not remove dental filling materials. Amalgams are easy to detect and to pry loose from the tooth. Composite resin filling materials are usually not opaque on X-rays and often difficult to detect. Failure to detect and to remove them prior to processing to paraffin wax can result in considerable damage to the microtome knife.

Fixation, dehydration, and embedding will take longer, as tissues are denser and reagents penetrate at slower rates.

Sectioning is difficult, as the knife has to pass through a series of layers, each of which differs in hardness. Sectioning is gen-

\section{Continued on following page}

\section{Si (Li)-Detectors}

stainless steel dewar, UHV technology excellent energy resolution best choice for light element analysis unrivalled long term stability

\section{Microanalysis Systems}

standardless andysis on rough surfaces and particles physical calculation of the bremsstrahlung integrated vexpert system « comprehensive error calculation application-specific automatic quantification

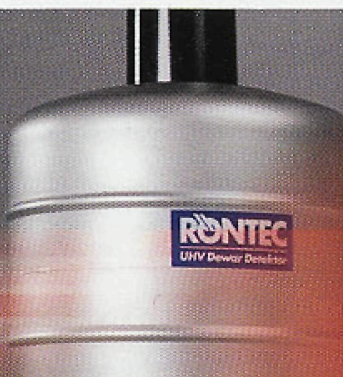

\section{NEW! atement Imaging}

liquid nitrogen free RÖNTEC XFlash detector 8 times faster than EDX with digital pulse processing. combines with any existing EDX 50
keps or imaging system

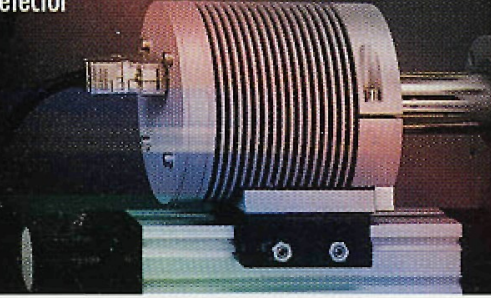

\section{Perfection that fascinates}

-




\section{Teeth and their Associated Tissues Continued from preceding page}

erally best if slow and if the direction of cut is carefully selected to allow a gradual introduction to the most dense regions. Compression in one direction is a common artifact. Passage of the knife through layers that vary considerably in their density and hardness also often results in chatter.

There is a tendency for the collagen fibers in the dentin to swell when sections are floated on a water bath, so when the section dries, folds form in the dentin and often in the surrounding bone. Staining of these areas then occurs from both sides of the section with a resulting darker stain in these areas and sometimes stain precipitation. These can be minimized or eliminated by carefully brushing the dentin with a fine paintbrush. The process is repeated until the hard tissue has dried, usually within a few minutes. This technique sounds crude but works.

Most of the standard staining techniques can be used on sections of bone and teeth but consideration needs to be given to the denser nature of the hard tissues and the slower rate of penetration of the reagents including the dyes. For general histological details, hematoxylin and eosin stain work well but best results are obtained when more dilute staining solutions than those for soft tissues are used. The use of very weak bluing and differentiating solutions is also recommended. Using these weaker staining solutions will accentuate growth and resting lines in the hard tissues. Even if using undiluted stain, more time will be required for staining hard tissues. A 5 minute van Gieson stain is necessary to uniformly stain the collagen fibers in dentin compared to 2 to 3 minutes for soft tissues. Sharpey's fibers embedded in the bone of the socket and the cementum of the root require an intense stain such as Heidenhain's Azan.

Adhesion of sections of hard tissue to the slide is a common problem especially with dentin, but can be improved by thinly coating the section of the slide with celloidin. Celloidin will, however, act as a barrier to enzymes and it will also permanently stain if certain dyes such as celestine blue are used.

Image analysis of teeth, implants and surrounding tissues is usually carried out using ground sections. With care, the amoun of shrinkage and distortion with these is kept to a minimum, compared to the use of paraffin sections.

These comments regarding the preparation of hard tissue outline some of the major problems that can be encountered. Workers that have produced a stained section of a tooth with the surrounding tissues will, however, attest not only to the skill that was necessary to produce a section without wrinkles and with even staining, but also the beauty and organization of the tissues.

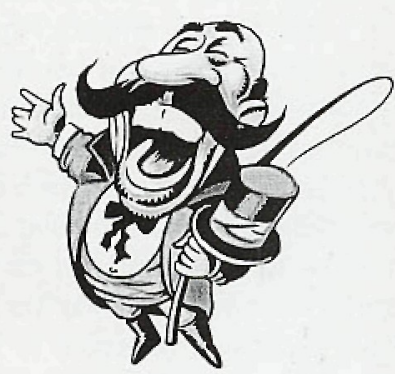

\section{Crystal Clear.}

\section{The Meiji EM Series of Modular Stereo Mitroseapes.}

If you are looking for precision, durability, quality and value in a stereo microscope, we invite you to take a closer look at Meiji's EM Series of Stereo Microscopes.

The modular design (A wide variety of bodies, single magnification or zoom - rotatable $360^{\circ}$, auxiliary lenses, eyepieces, stands, holders, etc.) gives you the freedom to create the ideal instrument for your specific needs or application, and Meiji stands behind every instrument with its limited Lifetime Warranty.

For more information on these economically priced stereo microscopes, please call, FAX or write us today.

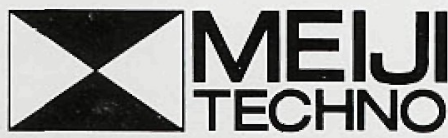

\section{MEIJI TECHNO AMERICA}

2186 Bering Drive, San Jose, CA 95131, Toll Free Telephone: 800.832 .0060 FAX: 408.428.0472, Tel: 408.428.9654 
